Meta

Journal des traducteurs

Translators' Journal

\title{
Le truchement
}

\section{Jacques Hassoun}

Volume 27, numéro 1, mars 1982

Psychanalyse et traduction

URI : https://id.erudit.org/iderudit/003039ar

DOI : https://doi.org/10.7202/003039ar

Aller au sommaire du numéro

Éditeur(s)

Les Presses de l'Université de Montréal

ISSN

0026-0452 (imprimé)

1492-1421 (numérique)

Découvrir la revue

Citer cet article

Hassoun, J. (1982). Le truchement. Meta, 27(1), 119-124.

https://doi.org/10.7202/003039ar

Ce document est protégé par la loi sur le droit d'auteur. L'utilisation des services d'Érudit (y compris la reproduction) est assujettie à sa politique d'utilisation que vous pouvez consulter en ligne.

https://apropos.erudit.org/fr/usagers/politique-dutilisation/
Cet article est diffusé et préservé par Érudit.

Érudit est un consortium interuniversitaire sans but lucratif composé de l’Université de Montréal, l'Université Laval et l'Université du Québec à Montréal. Il a pour mission la promotion et la valorisation de la recherche. https://www.erudit.org/fr/ 


\section{LE TRUCHEMENT}

JACQUES HASSOUN

Quand le voyageur, souvent un pélerin se rendant en Terre Sainte, débarquait dans l'un des États du Levant, il embauchait dans un des ports où son navire faisait escale, un homme de confiance; traducteur-interprète susceptible de lui simplifier les démarches à effectuer auprès des autorités locales, le truchement le suivait dans ses déplacements et à l'occasion le doublait. Ce mot truchement est en lui-même une redondance. Venu de l'italien dragomanno, du byzantin dragoumano $\xi$, le terme est sémitique : torgueman (traducteur, interprète). Ce n'est que tardivement qu'il signifie «ce (ou celui) qui fait comprendre».

Ainsi l'européen qui avançait péniblement de Gênes à Constantinople, à Rhodes, puis à Alexandrie, Jérusalem et Nazareth, se trouvait doublé d'un intermédiaire. Immuabilité du parcours - des centaines de chroniques, de la Renaissance à la fin du XIX ${ }^{\mathrm{e}}$ siècle, en font foi ${ }^{1}$-, immuabilité du cérémonial... Un truchement parfois membre d'une de ces minorités religieuses qui firent les beaux jours de l'Empire Ottoman suivait le pélerin. Ami, frère, importun, encombrant, et pour tout dire servant, on le voit dans les récits de voyage s'imposer à l'étranger au point de devenir indispensable. Des familles, des dynasties d'accompagnateurs s'instituèrent dans cette fonction, au point qu'en divers points de l'Empire soumis à la Sublime Porte apparurent les patronymes de Torgueman ou Tordjman...

Un pieux voyageur souhaitait-il accomplir tel parcours qui ne convenait pas au truchement? Aussitôt celui-ci invoquait la présence de tribus de barbares vivant de rançons, anthropophages, adorateurs de divinités fabuleuses, censées sévir dans la région à éviter, et propres à rebuter le plus courageux, propres aussi à créer une anthropologie fantaisiste... Tel autre rêvait-il de quelque courtisane, de quelque visite scandaleuse dans un harem? La mise en scène féerique aussitôt surgissait, tous les interdits locaux apparemment abolis devant les vertus persuasives d'un truchement roublard, malin et menteur. Que de bordels levantins furent décrétés demeures de mamelouks sanguinaires prêts à surgir pour étriper l'adorateur du Nazaréen, qu'importe? La magie verbeuse du truchement - appelé aussi drogman jusqu'à nos jours dans les Consulats de France du Proche-Orient - agissait avec efficience. Le truchement ne se con-

1. Cf. Jean Thevenot (1645): Voyage de Levant, réédité chez F. Maspéro, (1980); Joos van Ghistele (1482-1483): Le voyage en Égypte, réédité par l'Institut français d'Archéologie Orientale du Caire (1976); Gérard de Nerval: Voyage en Orient, réédité par Julliard (1966); Eugène Boullier (1856): Journal d'un voyage en Orient, Godbert à Laval. 
tentait pas de traduire les dialogues. Il traduisait le paysage au point de le rendre vrai. Que serait d'ailleurs un paysage qui ne passerait pas par le discours, sinon un objet à conquérir ou à détruire, sinon une nature, non plus morte (c'est-à-dire retranscrite), mais cadavérique, obscène, offerte. Il traduisait les usages jusqu'à les rendre vraisemblables. Il traduisait la géographie - ô sources du Nil que de belles fantasmagories ont-elles été écrites en ton nom! - jusqu'à la rendre compréhensible aux Barbares venus d'Outre-Méditerranée.

Car somme toute ce que le truchement et le pélerin construisaient de concert était le donné-à-voir de l'un, interprété au profit d'un autre... qui en rendait compte à des tiers. Ou qui serait susceptible de le faire : le truchement fonde une vérité : celle qui procéderait d'une rencontre dans ce que celle-ci a de hasardeux et de fatal tout à la fois et d'où le trop-bien-entendu est à peu près exclu.

N'est-ce pas dans ce que ces chroniques ont de plus étrange pour nous aujourd'hui, que nous pouvons apprécier à quelle vérité subjective pélerins et truchements s'abandonnaient avec passion? D'ailleurs quand l'historien en arrive à écrire l'histoire à partir de ces chroniques, mais aussi à partir de documents réputés être indiscutables quant à leur véracité - actes notariés ou archives - ne lui arrive-t-il pas de se heurter à la question du sujet. Que métaphoriserait celui qui interprète et dit sa vérité.

Si Hérédote a survécu au point de nous être familier encore aujourd'hui, si Benjamin de Tudèle ou Joseph Cattaoui-Sambari qui à côté de belles et véridiques chroniques, parlent - et après eux des générations de pieux voyageurs - de fleuves fabuleux qui s'arrêtent de couler le samedi, si tant et tant de récits de voyages rapportent immuablement les mêmes fabliaux et les mêmes charmants tableaux baroques surchargés d'images, à peine différents les uns des autres, n'est-ce pas encore notre truchement qui est en cause?

Le truchement garant du voyage, vous l'avez deviné, occupe une place tout à fait particulière dans ces presque-réalisations de désirs que furent souvent ces pieuses péripéties, tenant lieux d'épopées fabuleuses et de chevauchées héroïques. Le truchement est le sancho-pança de ces abbés don-quichotesques, de ces pieux nobliaux en mal de croisades, de ces bourgeois confits en dévotion... qui n'hésitaient pas au décours d'une envolée mystique à signaler l'intérêt qu'aurait leur bonne ville de Gand ou de Lyon à établir quelques comptoirs dans ces villes du Levant, même si pour ce faire, ils se devaient de payer taxes et pots de vin à des percepteurs juifs ou à des schismatiques marchands de soie ou d'épices.

Car le truchement ne se faisait pas faute d'organiser de telles rencontres. Certes la foi de nos pieux touristes en sortait exaltée, tant leur semblaient ridicules les croyances de ces infidèles, mais le doute s'insinuait en eux. Créant de la surprise et parfois de l'étonnement. Ici des pré-chalcédoniens-coptes avaient quelque accent touchant, en évoquant le mystère de la transubstantation, propre à insinuer le doute dans la linéarité des illusions de l'européen, là des comportements judaïques incompréhensibles tant ils semblaient non-ghéttoïques, ébranlaient quelque peu des croyances en certaines psychopathologies de la vie quotidienne de ces parias, au point de leur donner, scandaleusement, un visage quelque peu humain. 
Provocateur, le truchement ne peut que l'être. N'est-il pas par son polyglottisme affairé à la limite de ce qu'il peut s'autoriser comme écarts? Car de cette limite sur laquelle il se situe, il ne peut qu'en jouer. Il n'est déjà plus là où il vit, et il ne vit que de ne pas en partir. Il est présent avec son savoir approximatif, sa connaissance trébuchante d'une multiplicité de langues, d'idiomes et de dialectes, là où le voyageur qui débarque arrive avec sa curiosité, mais aussi avec ses illusions. Nourries par ceux-là mêmes qui l'ont précédé.

Pas de truchement sans voyageurs, pas de visiteurs sans truchement et c'est à ce titre que celui-ci est comme le représentant. Un représentant qui ne peut soutenir son rôle qu'à se défaire d'une part du vrai sur le vrai, pour permettre à ceux qui viendraient avec l'espoir toujours déjoué de participer d'une histoire dont ils ne peuvent être les acteurs qu'en en reconnaissant la part qui leur échappe, de circuler. C'est bien parce que le truchement met une parole là où il y a de l'impossible à voir sinon à imaginer ou de l'incomplétude dans ce qui relève de la seule donnée des sens, qu'il joue son rôle. Qui ne saurait être celui d'une machine-traductrice qui se contenterait de débiter de la signification, c'est-à-dire somme toute de l'identique.

Mais avant que de nous engager dans cette voie plus avant, retournons vers notre truchement qui n'est intermédiaire qu'à jouer du désir - dans ce que celui-ci suppose comme ignorance quant à son objet - d'au moins deux personnes qui ignoreraient qu'il ne saurait y avoir de coïncidence entre leurs projets. Mais ce qui apparemment coïnciderait grâce au truchement de notre drogman passe par une parole - la sienne - où se négocierait la demande de l'un et le désir de l'autre...

Mais imaginons, imaginons notre drogman de retour dans ses foyers. Sa langue s'est enrichie de quelques mots que le puriste dirait étrangers. Mots concernant la nourriture et l'art de l'accommoder, exclamations de rage ou de dégoût, mots de pérégrinations aventureuses et de marchandages hasardeux, vont former cette langue franque qui permettra aux uns et aux autres de s'entendre. Dans le clair-obscur des venelles où se marchandent les trouvailles. Objets insolites ou chair dont la fraîcheur serait mesurable à l'aune d'un bagoût menteur. Cependant que substantifs, verbes, qualificatifs, vont par infections de proche en proche, envahir la langue indigène qui ne s'en remettra pas.

Qui douterait aujourd'hui que ce même phénomène n'ait pas gagné la langue des visiteurs? Que les uns et les autres puissent se parler, s'entendre, entrer en commerce on en déduit, n'est-ce pas la mise en échec, la faillite de cette monstruosité d'adéquation que serait la communication? La coïncidence qu'introduit le truchement serait celle qui obéirait aux lois logiques du hasard et non à celle qui tendrait à faire du $U n$, celle qui ferait que deux choses s'emboiteraient au point de ne plus faire que signe.

Et c'est ainsi que nous pouvons commencer à percevoir le rôle que joue, que nous faisons jouer à notre truchement: il serait celui qui viendrait infirmer les thèses saussuriennes, puisque désormais il n'est de langue maternelle... qu'étrangère. Langue où l'Autre n'est pas sans être présent. 
Leila militante d'extrême-gauche vit exilée à Paris. Elle a fui son pays natal après avoir été impliquée dans quelques activités dites subversives qui ont très vite tourné court.

Usant d'un français châtié dans la vie courante, c'est en cette langue qu'elle va faire son analyse, même si son analyste - malgré les différences dialectales - n'ignore pas la langue que Leila a parlé au cours de sa première enfance. Le choix même de son analyste, avait-elle dit au cours de son premier entretien, impliquait la mise en jeu de cette communauté de langue, mais aussi de différents autres éléments comme l'appartenance de son analyste à telle minorité nationale pour qui elle avait grande sympathie, ainsi que l'expérience partagée de l'exil.

Or, au cours d'une séance, rapportant un souvenir qui avait toutes les tonalités d'un plan fixe projeté sur un écran, elle décrit un des membres de sa famille, qui au jour de l'Indépendance de son pays, se trouvait assis par terre, seul. Grand seigneur tribal brisé d'avoir trop parié sur la puissance tutélaire... «il était assis», dit-elle, «sur la...»Ici, sa mémoire trébuche. Elle ne trouve plus le mot français. Après avoir beaucoup hésité elle le murmure dans sa langue maternelle : «ataba».

Le mot autant que la scène arrête l'attention de son analyste, qui ne pipe mot. À la séance suivante, elle revient sur cette scène qui lui évoque certaines de ses difficultés actuelles, puisqu'elle se trouve vivre sur le territoire de l'ancienne puissance protectrice pour avoir été plus radicale que les plus acharnés des indépendantistes de son pays. Puis le mot dont elle a oublié la traduction française revient avec insistance. Elle s'irrite de ne pouvoir le dire en français. C'est alors que l'analyste s'autorise à lui en donner la traduction. D'autant que ce mot il le connaissait bien. N'avait-il pas même traduit une comptine où ce mot figure? Contre sa propre attente, il s'entend dire «... l'entrée...». Leila se fâche : "Non... ce n'est pas ça...» En effet, l'analyste s'était entruché ${ }^{2}$. Aussi reprenant la parole, il précisera que ce qui semblait être une traduction n'était qu'une approximation. Ce mot signifie le seuil, le pas de la porte, ou la marche qui dans les maisons orientales précède le seuil, ou sépare une pièce de l'autre.

L'hésitation qui apparait dans cette intervention apparemment faite dans le but de traduire un terme que son analyste ne connaissait que trop bien, ne fut pas sans permettre la mobilisation d'une chaîne associative insoupçonnable, dont les développements n'ont pas à figurer ici. Sinon d'ailleurs que l'ensemble «entrée» / «le pas de la porte» - qui sépare - / «le seuil»/ «pas de porte» (somme à payer pour entrer dans la jouissance d'un lieu) n'apparut à l'analyste comme une intervention faisant jeu de mot et truchement que dans un temps second: quand il eut à écrire ce fragment d'analyse afin de le consigner dans un texte promis à la lecture d'au moins un (e tierce personne).

2. S'entrucher: (terme du dialecte lorrain) s'étrangler. Exprime aussi l'idée de «faire une fausse route, par l'emprunt d'un passage inadéquat». La notion de passage dans cette expression étant centrale quant à ce qu'elle exprime (cette expression m'a été rapportée par MarieAnge Cabasso). 
Ceci pose différentes questions. Et d'abord celle de l'intervention de l'analyste, qui suppose toujours un temps de vacillement, mais aussi d'émergence de ce qui abolit le sens.

L'intervention métaphorise et à ce titre, elle ne saurait fournir de la signification. Ou tout au moins (comme les séances suivantes de ce fragment d'analyse le donnèrent à entendre), ce qui aurait pu se réduire à de la signification, devint signifiant. Aussi, ce fragment d'analyse n'est intéressant qu'à rendre compte de ceci : que le transfert en ouvre ne peut qu'interdire la paraphrase traductrice, le pareil au même d'une langue à l'autre.

L'analyse se joue de - et dans - l'équivoque dans ce qui pourrait à la limite être un malentendu.

L'interprétation se joue dans un mi-dire, qui en est l'enjeu. En un seuil qui serait le lieu d'un non-lieu, espace d'un montage, d'une construction qui se jouerait du réel. Point de nouage qui fonderait la vérité du sujet.

Ceci rendrait-il compte de ces analyses - nombreuses - qui se déroulent comme par nécessité dans une autre langue que la maternelle? Poser cette question, n'est-ce pas une manière de rendre compte que lorsque $J e$ parle, $l l$ ne se traduit pas, $l l$ ne traduit pas.

$J e$ parle hors toute traduction, même dans le baragouinage le plus infâme, sa propre vérité ${ }^{3}$. C'est peut-être ce qui rend compte que dans l'après-coup de l'analyse, ou en ces temps de passage, de passe, qui se présentent en fin d'analyse, il en est qui du lieu de leur propre histoire, découvrent que langues étrangères a pu s'inscrire comme signifiant dans les générations précédentes. Comment ces analysants pourraient-ils en entendre l'impact si l'analyse ne leur avait pas laissé percevoir ce qui de la trace s'efface pour signifier? Comment pourraient-ils les entendre si la per-laboration et la construction dans l'analyse ne venaient pas parler le cinéma muet de la réminescence?

Muet à force de traduire sans arrêt des flots d'images... à moins que l'analyste à l'instar du truchement ne vienne y mettre un terme : celui du sujetsupposé-savoir.

Car le truchement - comme fonction - nous l'avons vu, donne la parole à cette part de l'image qui ne peut que se déduire d'un discours, jusqu'à en instituer la vérité.

Et d'ailleurs théoriser, n'est-ce pas user d'un truchement? N'est-ce pas aussi rendre compte que l'analyste tel qu'il est pris dans la tâche ne peut que se situer dans un à-côté approximatif, et que c'est seulement à ce prix qu'une analyse peut se dérouler? Hors du duel, dans un écart qui permet à l'après-coup de l'analyse de se formuler en termes de théorisation et non d'acting-out mortifères.

3. Méme et surtout quand de par la grâce de la Sainte Inquisition (de quelque religion qu'elle soit d'ailleurs) ou par un souci de conformité cependant que des Zvi deviennent ici des Hirsch, là des Herschbert et ailleurs des Cerf, et que des Aboulafitolédans se convertissent en de la Fuerza, et que des Cohen se réveillent un matin affublés du patronyme de Sacerdoti à Venise, Santa-Cruz à Cordoue... et Van San-Croos à Amsterdam, le nom patronymique, lui, résiste irréductiblement à la traduction. Il peut devenir pourtant un private-joke ou un Witz qui permet la survie ou qui serait convoqué à cet effet. 
L'expérience des dernières années n'a-t-elle pas montré le poids que des auditeurs ont pu donner aux discours théoriques de quelques-uns au point de créer - fait nouveau dans l'histoire du mouvement analytique - un phénomène de transfert à l'analyse (ou à la théorie analytique)? Aujourd'hui, en France, au temps d'une après-règle fertile en rebondissements institutionnels, le milieu analytique semble au mieux ne plus être capable que de lancer quelques slogans dont le moins dramatique ou le moins comique serait: "du truchement! du truchement!» comme d'autres crieraient «de l'Autre! de l'Autre!» ou «un conte! un conte $! » . .{ }^{4}$ Ce qui n'enlève rien à la nécessité de théoriser... au contraire.

Car enfin, l'auteur de ces lignes adressées Outre-Atlantique a connu sur un mode mineur cette expérience : il n'a vraiment entendu ce qu'il élaborait en termes de «langue maternelle» qu'à travers le truchement de son traducteur occasionnel - Thomas Gora - à un séminaire organisé à New York.

La traduction de l'intraduisible du jeu de mot, n'est-ce pas la tâche à laquelle le truchement s'attache?

N'est-ce pas ce à quoi l'analyste doit quotidiennement s'affronter?

4. Et au pire, de créer des dynasties de Forgueman : je veux parler ici du label en passe de devenir signe patronymique : le label lacan. 